\title{
Tratamiento neoadyuvante con imatinib de un tumor gigante del estroma gastrointestinal rectal
}

\author{
Neoadjuvant treatment with imatinib for a giant tumor of the rectal \\ gastrointestinal stroma \\ Javier Pérez-Calvo1', Jordi Castellví-Valls², Lorenzo Viso-Pons¹, Luis Ortiz-de Zárate ${ }^{1}$, \\ Verónica González-Santin", Patrizio Petrone ${ }^{3}$ \\ 1 Servicio de Cirugía General y del Aparato Digestivo, Hospital de Sant Joan Despí Moisès Broggi, Consorci Sanitari Integral, \\ Barcelona, España \\ 2 Jefe de Servicio de Cirugía General y del Aparato Digestivo, Hospital de Sant Joan Despí Moisès Broggi, Consorci Sanitari Integral, \\ Barcelona, España \\ 3 Director of Surgical Research, Department of Surgery, NYU Winthrop Hospital; Mineola, Long Island, Nueva York, USA; Clinical \\ Associate Professor of Surgery, New York Medical College; Valhalla, New York, USA
}

Poster presentado en la XXI Reunión Nacional de Cirugía de la Asociación Española de Cirujanos en Málaga, España, octubre de 2017

\section{Resumen}

Alrededor de $5 \%$ de los tumores del estroma gastrointestinal (GIST) se localizan en el recto. Cuando se encuentran localmente avanzados, el tratamiento neoadyuvante con imatinib ha demostrado buenos resultados para reducir el volumen de este tipo de tumores.

Se presenta el caso de un paciente con diagnóstico de GIST rectal gigante, al que se le administró neoadyuvancia con imatinib y, posteriormente, se sometió a resección anterior baja con anastomosis coloanal.

Es imprescindible que la evaluación y el tratamiento sean multidisciplinarios en los GIST rectales, para tratar de obtener los mejores resultados ante esta entidad tan poco frecuente, poder evitar la comorbilidad asociada y practicar cirugías menos agresivas tras una buena reacción terapéutica al imatinib.

Palabras clave: neoplasias del recto; tumores del estroma gastrointestinal; diagnóstico diferencial; oncología quirúrgica; terapia neoadyuvante; mesilato de imatinib.

\begin{abstract}
Less than $5 \%$ of gastrointestinal stromal tumors (GIST) are located at the rectum. When these tumors are locally advanced, neoadjuvant therapy with imatinib has shown good results, reducing its volume. We present the case of a patient with a giant rectal GIST tumor, who underwent neoadjuvant imatinib therapy, and posterior low anterior resection with coloanal anastomosis.
\end{abstract}

Fecha de recibido: 21/01/2018 - Fecha aceptación: 14/02/2018

Correspondencia: Patrizio Petrone, MD, MPH, MHSA, FACS. Director of Surgical Research, Program Director, International Research Fellowship, Department of Surgery-NYU Winthrop Hospital, 222 Station Plaza North, Suite 603, Mineola, Long Island, New York 11501, USA Correo electrónico: patrizio.petrone@gmail.com - ppetrone@nyuwinthrop.org

Citar: Pérez-Calvo J, Castellví-Valls J, Viso-Pons L, Ortiz-de Zárate L, González V, Petrone P. Tratamiento neoadyuvante con imatinib en un tumor gigante del estroma gastrointestinal rectal. Rev Colomb Cir. 2018;33:428-32. https://doi.org/10.30944/20117582.90

Este es un artículo de acceso abierto bajo una Licencia Creative Commons - BY-NC-ND https://creativecommons.org/licenses/by-nc-nd/4.0/deed.es 
In rectal GIST tumors it is essential the multidisciplinary evaluation and treatment, in order to obtain the best possible results in this rare entity. After a good response to the treatment with imatinib, aggressive surgeries can be avoided, along with the associated morbidity that comes with it.

Key words: rectal neoplasms; gastrointestinal stromal tumors; diagnosis, differential; surgical oncology; neoadjuvant therapy; imatinib mesylate.

\section{Introducción}

La incidencia de los tumores del estroma gastrointestinal (GIST) es muy baja, alrededor de Io a 20 casos por millón por año ${ }^{\mathrm{I}}$, de los cuales menos del $5 \%$ se localizan en el recto ${ }^{2}$. Cuando estos tumores se encuentran localmente avanzados, el tratamiento neoadyuvante con imatinib ha demostrado buenos resultados para reducir su volumen.

Se presenta el caso de un paciente con diagnóstico de un GIST rectal gigante, al que se le administró neoadyuvancia con imatinib, y después se sometió a una resección anterior baja con anastomosis coloanal.

\section{Caso clínico}

Se trata de un paciente varón, de 45 años de edad, con antecedentes familiares de cáncer de colon, que acudió a urgencias, derivado de otro centro hospitalario por proctalgia y sensación de ocupación pélvica de 15 días de evolución. En aquel momento, el diagnóstico fue orientado como un absceso perianal. Se hizo una exploración anal quirúrgica, en la cual se observó una gran masa rectal. Se tomaron biopsias, las cuales no fueron concluyentes, y el paciente fue derivado a nuestro hospital.

A su arribo, el paciente refirió aumento de la intensidad de la proctalgia, sensación de ocupación pélvica y síndrome tóxico asociado. Presentó ligera incomodidad a la palpación en la fosa ilíaca izquierda. Se percibió una tumoración submucosa pétrea al tacto rectal, ubicada a $3 \mathrm{~cm}$ del margen anal, en contacto con el músculo puborrectal.

Ante estos hallazgos, se practicó una tomografía computadorizada (TC) abdominal en la que se observó una masa rectal de $73 \times 62 \times 48 \mathrm{~mm}$ (figura I), sin demostrar una clara afectación de la grasa perirrectal, y sin que se identificara un plano graso de separación del suelo prostático. No se observaron ganglios linfáticos sospechosos.

$\mathrm{Al}$ no poder demostrarse la afectación de tejidos y órganos vecinos, se decidió practicar una resonancia magnética (RM) pélvica (figura 2), la cual no mostró una clara infiltración de la grasa mesorrectal, aunque sí un borramiento en el lado izquierdo del recto, sugerente de tumoración de tipo GIST.

Posteriormente, en una colonoscopia se encontró subestenosis de la luz rectal debida a la mucosa con edema y eritema observándose normal el sector más proximal. Se tomó una biopsia quirúrgica debido al bajo rendimiento diagnóstico de la biopsia endoscópica de este tumor (33\%), ya que no se pudieron obtener muestras del tejido más profundo con las pinzas

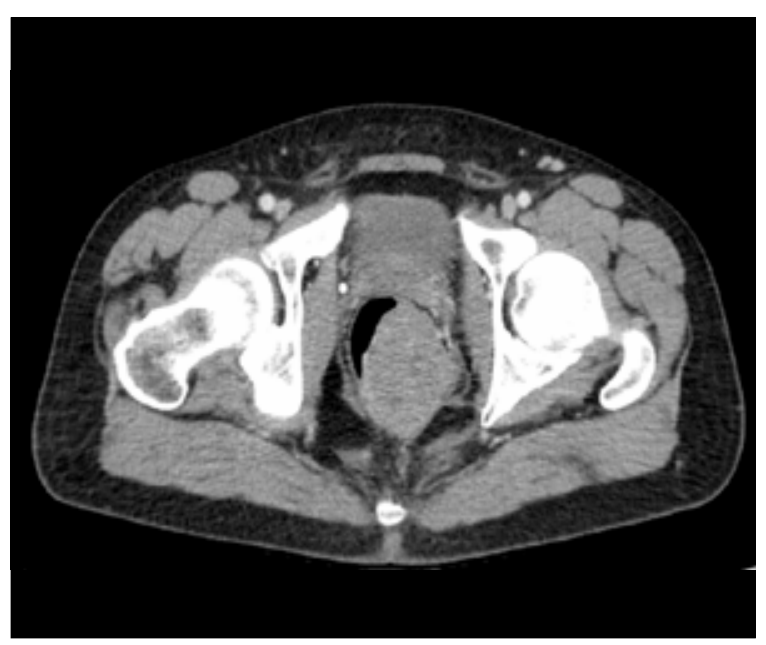

Figura 1. TC abdominal en la que se observa masa rectal de 73 × 62 × $48 \mathrm{~mm}$, sin una clara afectación de la grasa perirrectal, y sin identificarse un plano graso de separación del suelo prostático. 


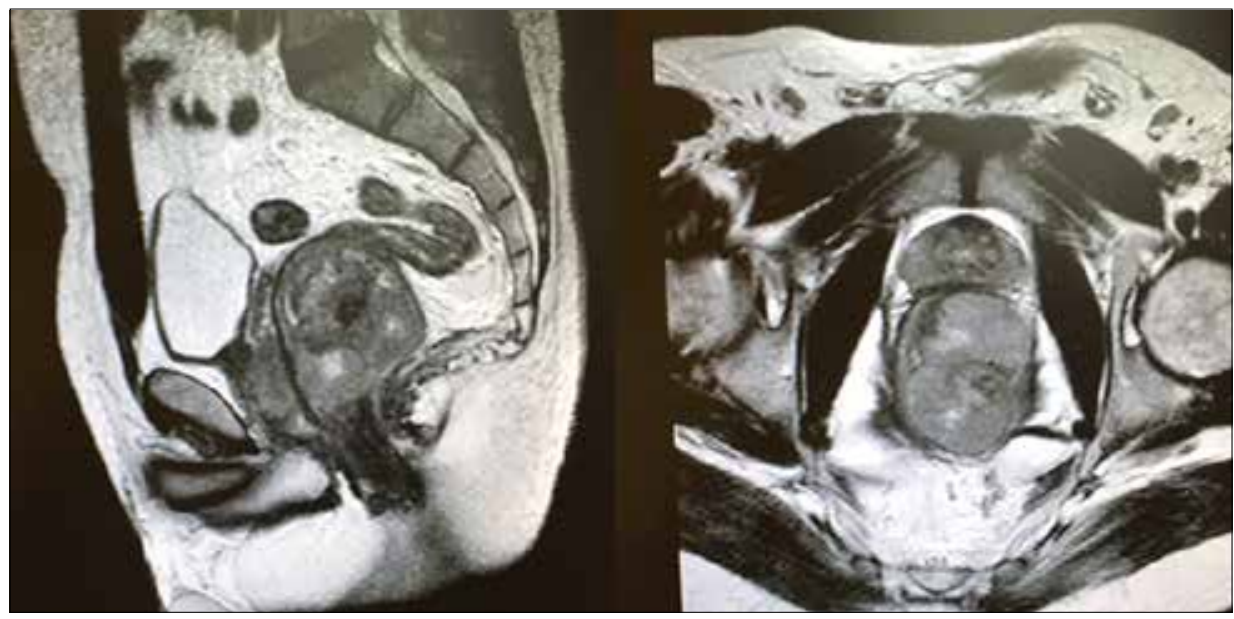

Figura 2. RM pélvica, en la que se observa borramiento en el lateral rectal izquierdo, sugestiva de un GIST.

de biopsia endoscópicas habituales. El resultado anatomopatológico obtenido fue indicativo de un tumor del estroma gastrointestinal (GIST) de alto riesgo.

Finalmente, con los hallazgos clínicos, radiológicos y anatomopatológicos reunidos, se presentó en el Comité de Tumores Estromales, en el cual se decidió dar un tratamiento neoadyuvante con imatinib, con la intención de obtener una buena reacción y evitar una amputación abdominoperineal en este paciente. Se sometió a siete meses de neoadyuvancia acompañada de controles radiológicos periódicos. En el último control, se observó una disminución considerable del volumen tumoral, quedando este por encima de la reflexión peritoneal (figura 3). Se presentó nuevamente en el Comité con los hallazgos actualizados y se decidió indicar un tratamiento quirúrgico.

Debido al tamaño y la localización del tumor, se llevó a cabo una resección anterior ultrabaja por vía abierta, con anastomosis coloanal e ileostomía de protección. La pieza quirúrgica presentó márgenes libres de tumor. El paciente fue dado de alta al octavo día, sin complicaciones posoperatorias. El reporte de la anatomía patológica definitiva informó focos microscópicos residuales de tumor del estroma gastrointestinal (figura 4).

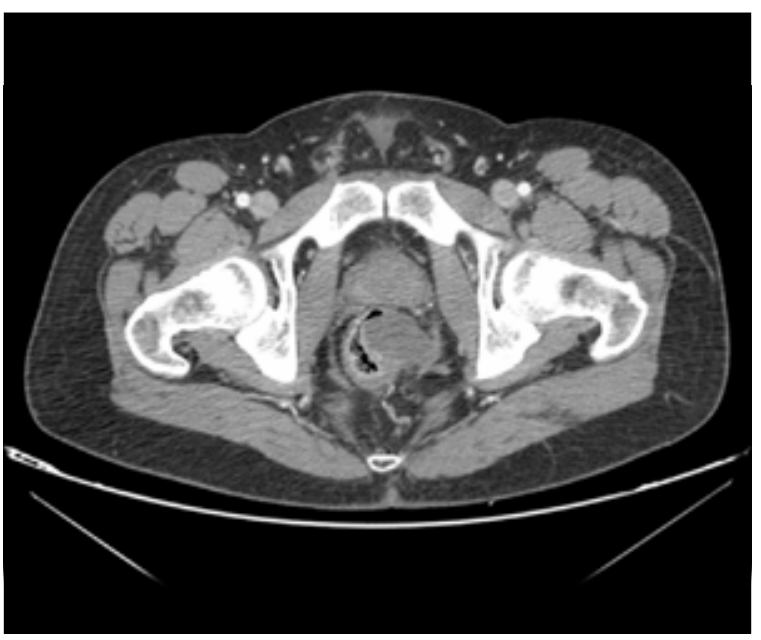

Figura 3. Disminución considerable del volumen tumoral después de la administración de imatinib, quedando el tumor por encima de la reflexión peritoneal.

La ileostomía se cerró I2 meses más tarde. Hasta el último control, I8 meses después de la cirugía, el paciente no presentaba signos de recidiva.

\section{Discusión}

Los tumores GIST son poco frecuentes, corresponden, aproximadamente, a I \% de los tumores del tubo digestivo; entre ellos, los rectales son aún más raros $(5 \%)^{3}$. Este tipo de tumores ha- 


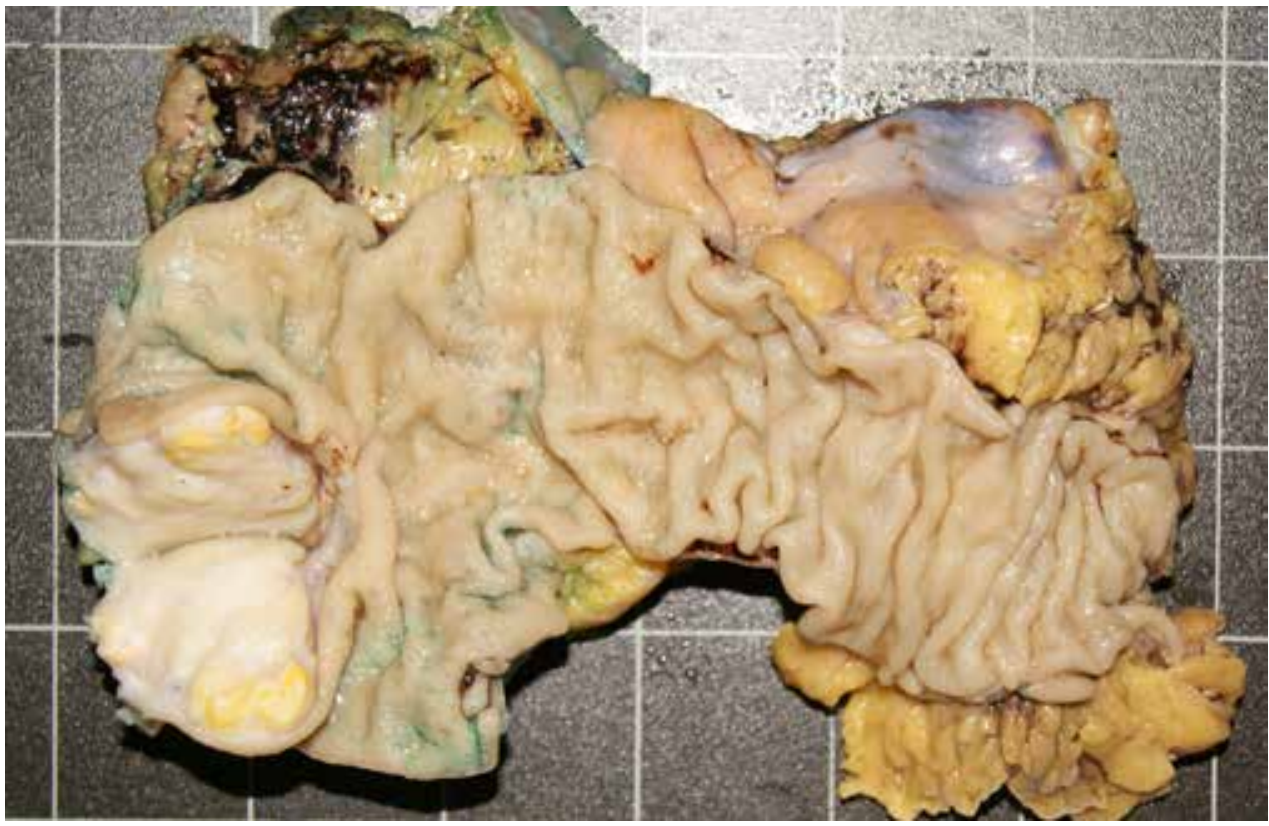

Figura 4. Pieza de anatomía patológica en el que se observa resección de recto, con focos microscópicos residuales de tumor del estroma gastrointestinal.

bitualmente son de gran volumen y se localizan en la pelvis, por lo que su manejo debe ser multidisciplinario.

Por su localización, y debido a que suelen estar íntimamente adheridos al suelo pélvico, el tratamiento quirúrgico suele ser un reto para el cirujano, ya que podría conllevar resecciones multiviscerales. El tratamiento quirúrgico de elección depende tanto del tamaño del tumor como de otros factores: en caso de que el tumor no se encuentre adherido a órganos vecinos y presente una distancia suficiente del margen anal, el tratamiento de elección es una resección anterior baja con anastomosis coloanal. No suele ser necesaria una escisión total del mesorrecto, ya que las metástasis ganglionares son raras. Por otro lado, en caso de tratarse de un recto bajo, el tratamiento que se debería llevar a cabo sería una escisión abdominoperineal completa ${ }^{4}$.

El imatinib, un inhibidor de la tirosincinasa (TKI), se ha convertido en la primera línea de tratamiento para este tipo de tumores y, en la actualidad, nos permite practicar cirugías menos agresivas debido a la reducción del tamaño tumoral y de su actividad mitótica 5 . Se obtiene una buena reacción terapéutica en casi el $50 \%$ de los pacientes sometidos a esta terapia, lo que permite reducir la morbilidad quirúrgica (tanto funcional como neurológica) y realizar procedimientos menos agresivos, por ejemplo, la cirugía transanal mínimamente invasiva (TAMIS) ${ }^{6} \mathrm{o} \mathrm{la}$ cirugía endoscópica transanal (TEO/TEM) ${ }^{7}$, entre otros. En el presente caso, se optó por una resección anterior baja por la imposibilidad de utilizar estas técnicas, debido al tamaño y la localización por encima de la reflexión peritoneal del tumor.

A pesar de los múltiples artículos publicados en la literatura, no existe consenso acerca de la dosis y la duración de la neoadyuvancia con imatinib $^{8}$. Habitualmente, se mantiene durante tres a seis meses, con controles radiológicos periódicos ${ }^{9}$. En lo que sí parece haber consenso es sobre la mayor eficacia quirúrgica después de 6 a I2 meses de haber iniciado el tratamiento con imatinib ${ }^{\text {10 }}$, ya que ha demostrado aumentar la supervivencia libre de enfermedad en los pacientes con GIST rectal ${ }^{4}$. Respecto a la radioterapia, no 
ha sido comúnmente aceptada como parte del tratamiento multidisciplinario de los tumores del estroma gastrointestinal, sino que se ha utilizado como tratamiento paliativo. Sin embargo, recientemente se han publicado pequeñas series de casos que sugieren que la radioterapia es una opción válida para controlar la progresión local en los tumores quimiorresistentes, siendo esta una alternativa con intención curativa en el manejo del GIST " ${ }^{\text {I. }}$

\section{Conclusión}

Es imprescindible hacer una evaluación y un tratamiento multidisciplinario en los GIST rectales, como fue el caso del paciente aquí presentado, en el cual se obtuvo un buen resultado ante este tumor tan poco frecuente, al evitar la comorbilidad asociada y, así, practicar una cirugía menos agresiva tras una buena reacción al tratamiento.

\section{Referencias}

I. Beltrán MA, Valenzuela C, Díaz R, Haito Y, Larraín C. Tumores del estroma gastrointestinal del duodeno: revisión de la literatura científica actual con énfasis en el tratamiento. Rev Colomb Cir. 20I4;29:140-54.

2. Romero AE, Mesa OA, Melo MA, Chinchilla SI, Barajas PA. Gastric gastrointestinal stromal tumor with unusual skull metastasis. Rev Colomb Gastroenterol. 2OII;26:3II-5.
3. Cavnar MJ, Wang L, Balachandran VP, Antonescu CR, Tap WD, Keohan M, et al. Rectal gastrointestinal stromal tumor (GIST) in the era of imatinib: Organ preservation and improved oncologic outcome. Ann Surg Oncol. 2017;24:3972-80.

4. Tielen R, Verhoef C, van Coevorden F, Reyners AK, van der Graaf WT, Bonenkamp JJ, et al. Surgical management of rectal gastrointestinal stromal tumors. J Surg Oncol. 2013;107:320-3.

5. Wilkinson MJ, Fitzgerald JE, Strauss DC, Hayes AJ, Thomas JM, Messiou C, et al. Surgical treatment of gastrointestinal stromal tumour of the rectum in the era of imatinib. Br J Surg. 2015;IO2:965-7I.

6. Wachter N, Wörns MA, Dos Santos DP, Lang H, Huber T, Kneist W. Transanal minimally invasive surgery (TAMIS) approach for large juxta-anal gastrointestinal stromal tumour. J Minim Access Surg. 20I6;12:289-9I.

7. Eldamshety O, Metwally IH, Ghoneem E, Elkashef WF. Resection of rectal GIST using a novel technique: A report of two cases. Ecancermedicalscience. 2017;17:760.

8. López-López V, Fernández JÁ, Parrilla P. Utility of neoadjuvant therapy in rectal GIST. Rev Esp Enferm Dig. 20I7;IO9:534-5.

9. Bannura G, Gallardo C, Cornejo V. Neoadyuvancia con imatinib en el manejo de GIST gigante del tabique rectovaginal. Rev Chil Cir. 20I4;66:175-80.

Io. Joensuu H, Eriksson M, Sundby Hall K, Hartmann JT, Pink D, Schütte J, et al. One Vs. three years of adjuvant imatinib for operable gastrointestinal stromal tumor: A randomized trial. JAMA. 2012;307:1265-72.

II. Ozkan EE. Radiotherapy for gastrointestinal stromal tumors. Chin Med J (Engl). 2018;131:235-40. 